\title{
290.
}

\section{A DISCUSSION OF THE STURMIAN CONSTANTS FOR CUBIC AND QUARTIC EQUATIONS.}

[From the Quarterly Journal of Pure and Applied Mathematics, vol. Iv. (1861), pp. 7-12.]

For the cubic equation

$$
(a, b, c, d)(x, 1)^{3}=0
$$

the Sturmian Canstants (or leading coefficients of the Sturmian functions) are

$$
\begin{aligned}
& a, a, b^{2}-a c,-a^{2} d^{2}+6 a b c d-4 a c^{3}-4 b^{3} d+3 b^{2} c^{2}
\end{aligned}
$$

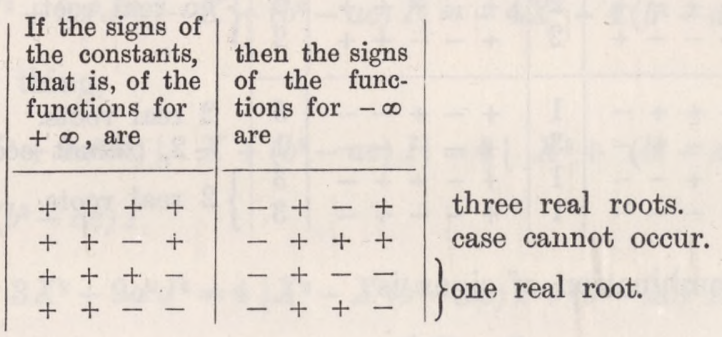

The second case would give a loss of variations of sign in passing from $\infty$ to $-\infty$, which is inconsistent with Sturm's theorem. To show a posteriori that the case cannot occur, we may form the identical equation

$$
\left(a^{2} d-3 a b c+2 b^{3}\right)^{2}=-a^{2}\left(-a^{2} d^{2}+6 a b c d-4 a c^{3}-4 b^{3} d+3 b^{2} c^{2}\right)+4\left(b^{2}-a c\right)^{3},
$$

and, this being so, then in the case in question, the right-hand side would consist of two terms, each of them negative, while the left-hand side is essentially positive.

C. IV. 
In the particular case where the third constant vanishes, or

we have

$$
b^{2}-a c=0
$$

$$
\begin{aligned}
& -a^{2} d^{2}+6 a b c d-4 a c^{3}-4 b^{3} d+3 b^{2} c^{2} \\
= & -(a d-b c)^{2}+4\left(b^{2}-a c\right)\left(c^{2}-b d\right) \\
= & -(a d-b c)^{2}, \text { is negative }
\end{aligned}
$$

hence, regarding the evanescent term as being at pleasure positive or negative, we have in each case a combination of signs corresponding to one real root.

The general result (which is well known) is, that there are three real roots or one real root according as

$$
-a^{2} d^{2}+6 a b c d-4 a c^{3}-4 b^{3} d+3 b^{2} c^{2}
$$

is positive or negative.

For the quartic equation

the Sturmian constants are

$$
(a, b, c, d, e)(x, 1)^{4}=0
$$

if, as usual,

$$
a, a, b^{2}-a c, 3 a J+2\left(b^{2}-a c\right) I, I^{3}-27 J^{2},
$$

$$
\begin{aligned}
& I=a e-4 b d+3 c^{2}, \\
& J=a c e-a d^{2}-b^{2} e+2 b c d-c^{3} .
\end{aligned}
$$

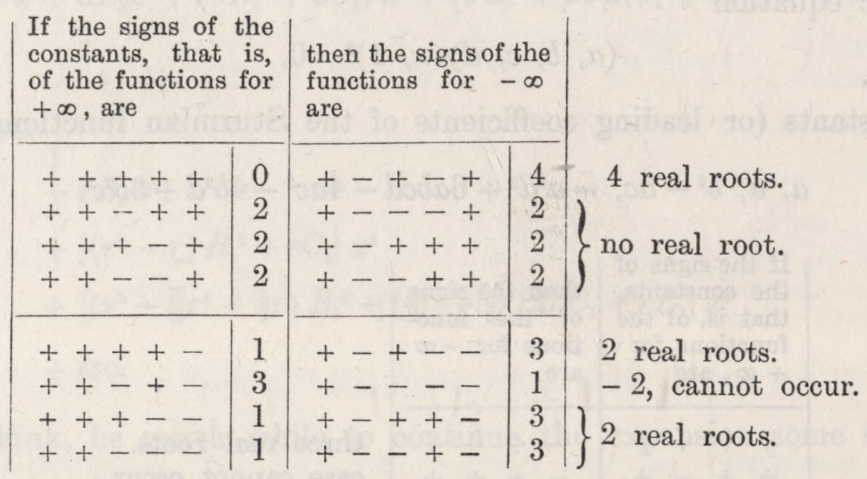

The non-existing combination of signs is

$$
\begin{aligned}
I^{3}-27 J^{2} & =- \\
3 a J+2\left(b^{2}-a c\right) I & =+ \\
b^{2}-a c & =-
\end{aligned}
$$

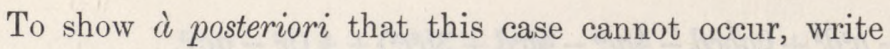

$$
\begin{aligned}
& 9=a^{2} d-3 a b c+2 b^{3}, \\
& X=3 a J+2\left(b^{2}-a c\right) I,
\end{aligned}
$$


then we have identically

$$
9\left(3 a^{2} J^{2}+X^{2}\right) 9^{2}=-4 a^{2} X^{3}+36\left(b^{2}-a c\right)^{3} X^{2}-4 a^{2}\left(b^{2}-a c\right)^{3}\left(I^{3}-27 J^{2}\right),
$$

which is impossible under the given combination of signs, since the left-hand side would be positive, and the right-hand side negative.

To prove the above identity-the relation $J U^{3}-I U^{2} H+4 H^{3}+\Phi^{2}=0$, between the covariants of the quartic, gives

$$
a^{3} J+a^{2}\left(b^{2}-a c\right) I-4\left(b^{2}-a c\right)^{3}+9^{2}=0,
$$

or, what is the same thing,

But

$$
\mathfrak{Q}^{2}=-a^{3} J-a^{2}\left(b^{2}-a c\right) I+4\left(b^{2}-a c\right)^{3} .
$$

and thence

$$
X=3 a J+2\left(b^{2}-a c\right) I
$$

$$
39^{2}+a^{2} X=-a^{2}\left(b^{2}-a c\right) I+12\left(b^{2}-a c\right)^{3},
$$

or

$$
39^{2}=-a^{2} X-a^{2}\left(b^{2}-a c\right) I+12\left(b^{2}-a c\right)^{3},
$$

and the identity will be true, if

$$
\begin{aligned}
\left(3 X^{2}+9 a^{2} J^{2}\right) & \left\{-X-\left(b^{2}-a c\right) I+12 \frac{\left(b^{2}-a c\right)^{3}}{a^{2}}\right\} \\
& =-4 X^{3}+36 \frac{\left(b^{2}-a c\right)^{3}}{a^{2}} X^{2}-4\left(b^{2}-a c\right)^{3}\left(I^{3}-27 J^{2}\right)
\end{aligned}
$$

This gives

$$
\left(3 X^{2}+9 a^{2} J^{2}\right)\left\{-X-\left(b^{2}-a c\right) I\right\}=-4 X^{3}-4\left(b^{2}-a c\right)^{3} I^{3},
$$

or, what is the same thing,

$$
\left(3 X^{2}+9 a^{2} J^{2}\right)\left\{X+\left(b^{2}-a c\right) I\right\}=4\left\{X^{3}+\left(b^{2}-a c\right)^{3} I^{3}\right\},
$$

or, dividing by $X+\left(b^{2}-a c\right) I$,

$$
3 X^{2}+9 a^{2} J^{2}=4\left\{X^{2}-X\left(b^{2}-a c\right) I+\left(b^{2}-a c\right)^{2} I^{2}\right\},
$$

and reducing

$$
X^{2}-4 X\left(b^{2}-a c\right) I-9 a^{2} J^{2}+4\left(b^{2}-a c\right) I^{2}=0,
$$

or finally

$$
\left\{X-3 a J-2\left(b^{2}-a c\right) I\right\}\left\{X+3 a J-2\left(b^{2}-a c\right) I\right\}=0,
$$

which is true in virtue of

$$
X=3 a J+2\left(b^{2}-a c\right) I
$$

and the identity is thus proved. 
The general conclusion is,

if

$$
I^{3}-27 J^{2} \text { is positive, the four roots are all real or all imaginary, }
$$

viz., all real if $b^{2}-a c$ and $3 a J+2\left(b^{2}-a c\right) I$ are both positive, imayinary if otherwise. But if $I^{3}-27 J^{2}$ is negative, the roots are two of them real, and the other two imaginary.

The following special cases may be noticed,

$$
1^{\circ} . \quad b^{2}-a c=0,
$$

here

$$
9\left(3 a^{2} J^{2}+X^{2}\right) \mathfrak{A}^{2}=-4 a^{2} X^{3}, \text { or } X=3 a J+2\left(b^{2}-a c\right) I=3 a J, \text { is negative, }
$$

so that,

if $I^{3}-27 J^{2}$ is + , the roots are all imaginary;

if $I^{3}-27 J^{2}$ is - , the roots are two real and two imaginary.

2. $X=3 a J+2\left(b^{2}-a c\right) I=0$,

here

$$
27 a^{2} J^{2 g^{2}}=-4 a^{2}\left(b^{2}-a c\right)^{3}\left(I^{3}-27 J^{2}\right)
$$

or $b^{2}-a c, I^{3}-27 J^{2}$ are of opposite signs, and if

$b^{2}-a c=-, I^{3}-27 J^{2}=+$, the roots are all imaginary,

$b^{2}-a c=+, I^{3}-27 J^{2}=-$, the roots are two real and two imaginary.

$3^{\circ} . \quad b^{2}-a c=0, X=3 a J+2\left(b^{2}-a c\right) I=0$,

here $J=0$, that is,

$2 b c d-a d^{2}-c^{3}=0$, or $(a d-b c)^{2}+c^{2}\left(a c-b^{2}\right)=0$, or $a d-b c=0$, and $I^{3}-27 J^{2}=I^{3}$,

$$
I=a e-4 b d+3 c^{2}=a e-4 \frac{b^{4}}{a^{2}}+3 \frac{b^{4}}{a^{2}}=a e-\frac{b^{4}}{a^{2}}=\frac{1}{a^{2}}\left(a^{3} e-b^{4}\right)
$$

whence

$$
I=+ \text {, the roots are all imaginary. }
$$

$I=-$, the roots are two real and two imaginary.

This is easily verified, in fact $a c-b^{2}=0, a d-b c=0$, give $c=\frac{b^{2}}{a}, d=\frac{b c}{a}=\frac{b^{3}}{a^{2}}$, and the equation becomes

$$
a x^{4}+4 b x^{3}+6 \frac{b^{2}}{a} x^{2}+4 \frac{b^{3}}{a^{2}} x+e=0
$$

or, which is the same thing,

$$
(a x+b)^{4}+\left(a^{3} e-b^{4}\right)=0,
$$

so that the roots are all imaginary, or two real and two imaginary, according to the sign of $a^{3} e-b^{4}$ as above. 
It may be noticed that for a quintic equation

$$
(a, b, c, d, e, f)(x, 1)^{5}
$$

if the Sturmian Constants are

$$
a, a, C, D, E, F \text {, }
$$

where as before $a$ is positive, then the roots are real or imaginary as follows: viz.,

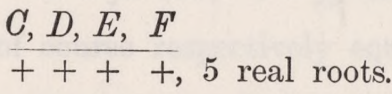

$$
\begin{aligned}
& \left.\begin{array}{l}
-++ \\
+-+ \\
--+ \\
++- \\
+-- \\
-
\end{array}\right\}+, 1 \text { real root, } 4 \text { imaginary roots. } \\
& \left.\begin{array}{l}
+++ \\
++- \\
+-- \\
--
\end{array}\right\}-, 3 \text { real roots, } 2 \text { imaginary roots. } \\
& -+-+ \text {, case which does not occur. } \\
& \left.\begin{array}{l}
-++ \\
+-+ \\
--+ \\
-+-
\end{array}\right\}-, \text { cases which do not occur. }
\end{aligned}
$$

The values of $C, D, E$, and $F$ are given in my "Tables of the Sturmian Functions for Equations of the Second, Third, Fourth, and Fifth Degrees," Phil. Trans., t. 147 (1857), pp. 733-736, [151], but I have not further examined this case.

2, Stone Buildings, W.C., September 29, 1859. 